\title{
Spin-Liquid States in a Copper-Based Material: The Mysterious Quantum Phase CuNCN
}

\author{
Andrei L. Tchougréeff ${ }^{1,2}$ and Richard Dronskowski ${ }^{1, *}$ \\ ${ }^{1}$ Institute of Inorganic Chemistry and Jülich-Aachen Research Alliance (JARA-HPC), RWTH Aachen University, \\ Landoltweg 1, D-52056 Aachen, Germany \\ ${ }^{2}$ Division of Electrochemistry, Department of Chemistry, Moscow State (Lomonossov) University and \\ Independent University of Moscow, Moscow Center for Continuous Mathematical Education, \\ Bolshoi Vlasevsky Per. 11, 119002 Moscow, Russia
}

\begin{abstract}
We review the many-body nature of the quantum material copper carbodiimide, CuNCN, which exhibits seemingly contradictive physical properties such as a local $\mathrm{Cu}(\mathrm{II})-\mathrm{N}$ coordination conforming to a 1st order Jahn-Teller effect, the total absence of magnetic neutron scattering and a fairly complex temperature dependence of its magnetic susceptibility indicating both Pauli- and Arrhenius-like regimes. It is shown that a spin-liquid (or resonating valence bond, RVB) approach for modelling the frustrated antiferromagnetic interactions in CuNCN not only allows for a vivid physicochemical picture of the compound but also predicts three RVB states differing in their dimensionalities as a function of the temperature. In addition, RVB theory semi-quantitatively describes subtle (and to a certain extent even paradoxical) structural effects in the lattice parameters at very low temperature. The alternative magnetically ordered or spin-Peierls models of CuNCN, however, are in conflict with the physical nature of the material.
\end{abstract}

\section{Keywords:}

\section{CONTENTS}

Acknowledgment

References and Notes

In its search for alternative materials, organometallic chemistry has provided several routes to novel compounds such as coordination polymers or "metal-organic" frameworks. ${ }^{1}$ Similarly, a highly successful solid-state approach has been to start with simple oxides but to formally replace the $\mathrm{O}^{2-}$ anion by complex quasi-molecular bridges containing nitrogen atoms, in the spirit of a "divalent" instead of a trivalent nitride anion. Within the last decade, we have predicted, synthesized and characterized the antiferromagnetic $3 d$ carbodiimides $^{2-6}$ incorporating $3 d$ cations connected by $\mathrm{NCN}^{2-}$ carbodiimide dianions as $\mathrm{O}^{2-}$ substitutes. These new $M \mathrm{NCN}$ species of the 1:1 stoichiometry adopt either rock-salt- or nickel-arsenide-like structures and may be regarded as chemical relatives of the correlated oxides. Also, the most recent 2:3 representative, $\mathrm{Cr}_{2}(\mathrm{NCN})_{3},{ }^{7}$ with a corundumlike structure is the first ferromagnetic carbodiimide with a Curie temperature of $178 \mathrm{~K}$, to be compared with the antiferromagnet $\mathrm{Cr}_{2} \mathrm{O}_{3}$. The physical properties of $3 d$ transition-metal carbodiimides are summarized in Table I.

*Author to whom correspondence should be addressed.
All $M$ NCN 1:1 compounds with $M=\mathrm{Mn}-\mathrm{Ni}$ represent a uniform family of optically transparent antiferromagnetic insulators, and these features are nicely reproduced with use of many-electron theory ${ }^{8,9}$ and also, with less certainty, by densityfunctional theory (DFT). By contrast, the copper compound $\mathrm{CuNCN}^{10}$ falls out of the row although its structure (Fig. 1) can be easily understood to originate from a 1st order Jahn-Teller distortion of the archetypical $M \mathrm{NCN}$ structure. Indeed, $\mathrm{CuNCN}$ poses an enormous challenge to (effective) one-particle theories such as DFT.

copper carbodiimide's physical properties are quite bizarre. CuNCN is a deeply black and semiconducting (but not metallic) material with an activation energy below $1 \mathrm{eV}$. Its magnetic properties are even more intriguing: as shown in Figure 2, the magnetic moments do not manifest themselves in the spinpolarized neutron scattering. ${ }^{10}$ The original susceptibility measurements as a function of temperature performed by SQUID magnetometry ${ }^{11}$ suggested quite a complex behavior with at least two magnetic transitions. These ideas have been refined using ESR spectroscopy, ${ }^{12}$ and the results are shown in Figure 3 which exhibit two distinct temperature regimes of the susceptibility: at higher-temperatures (above $80-100 \mathrm{~K}$ ) $\mathrm{CuNCN}$ is a temperatureindependent (Pauli-like) paramagnet which switches to a gapped (Arrhenius-like) paramagnet below $100 \mathrm{~K}$. Its activation energy (or gap) develops from zero to the low-temperature constant 
Table I. Crystal data and physical properties of the $3 d$ transition-metal carbodiimides.

\begin{tabular}{|c|c|c|c|c|c|c|}
\hline Formula: & $\mathrm{Cr}_{2}(\mathrm{NCN})_{3}$ & $\mathrm{MnNCN}$ & FeNCN & CoNCN & NiNCN & CuNCN \\
\hline Color: & Green & Green & Dark-Red & Orange-Brown & Light-Brown & Black \\
\hline Lattice parameters $(\AA ̊)$ : & $\begin{array}{c}a=5.57 \\
c=27.97\end{array}$ & $\begin{array}{c}a=3.36 \\
c=14.35\end{array}$ & $\begin{array}{l}a=3.28 \\
c=9.41\end{array}$ & $\begin{array}{l}a=3.21 \\
c=9.39\end{array}$ & $\begin{array}{l}a=3.15 \\
c=9.27\end{array}$ & $\begin{array}{l}a=2.99 \\
b=6.19 \\
c=9.41\end{array}$ \\
\hline Curie or Néel temperature $(K)$ & 175 & 28 & 345 & 255 & 360 & $?$ \\
\hline
\end{tabular}

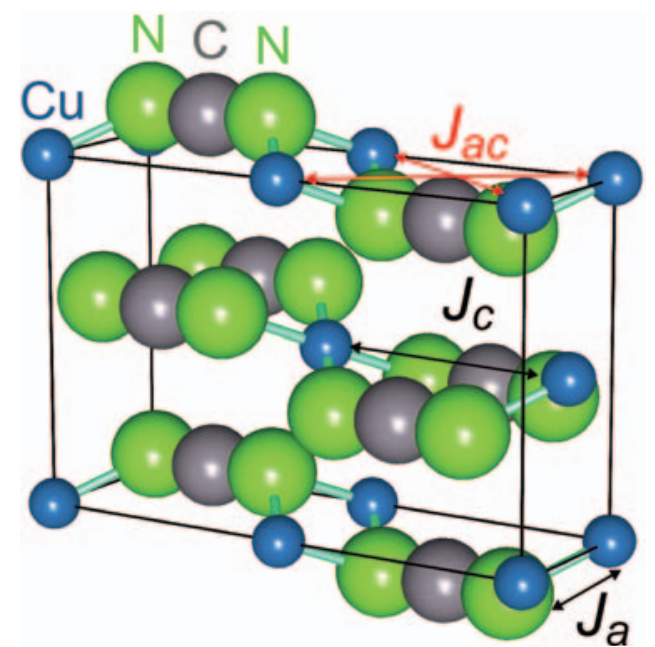

Fig. 1. The crystal structure of $\mathrm{CuNCN}$ and the exchange parameters in the crystalline ac plane. The magnetic model of the ac plane uses the $J_{c}, J_{a}$, and $J_{a c}$ interactions. Magnetic frustration appears when the interactions shown in red and black are simultaneously present; if interactions of only one type (red or black) are present, the corresponding magnetically ordered phases appear. Reprinted with permission of IOP from [13], A. L. Tchougréeff and R. Dronskowski, J. Phys.: Cond. Matt. 25, 435602 (2013). (c) 2013, All rights reserved. value as shown in the inset to Figure 3. Thus, seemingly contradictive items need to be reconciled which touch upon (i) the absence of the magnetic scattering and (ii) two (Pauli and Arrhenius) regimes of the course of the susceptibility as a function of temperature.

The aforementioned theoretical challenge can be mastered, however, by assuming a quantum spin-liquid (or resonating valence bond, RVB) phase of electrons/spins to occur in $\mathrm{CuNCN}$ and discarding simplistic one-electron models. Of course, the RVB states have been aware of in theoretical chemistry for more than a hundred years already, although often unconsciously so. We reiterate the well-known resonating valence-bond approximation for the electronic wave function of benzene as depicted in Figure 4. Because of the underlying symmetry, one can be sure that the weights/amplitudes of the two classical (Kekulé) structures and the three nonclassical (Dewar) structures (configurations) are the same such that a single parameter $(\varphi)$ suffices to completely describe the depicted RVB-type function. Let us therefore assume that the electrons (spins) in the $\pi$-system are described by an antiferromagnetic Heisenberg Hamiltonian. The double sticks seen in Figure 4 symbolize that the spins located on the corresponding atoms are coupled (paired) into the "valence bond state:"

$$
\frac{1}{\sqrt{2}}\left(c_{r \uparrow}^{+} c_{r^{\prime} \downarrow}^{+}+c_{r \downarrow}^{+} c_{r^{\prime} \uparrow}^{+}\right)
$$

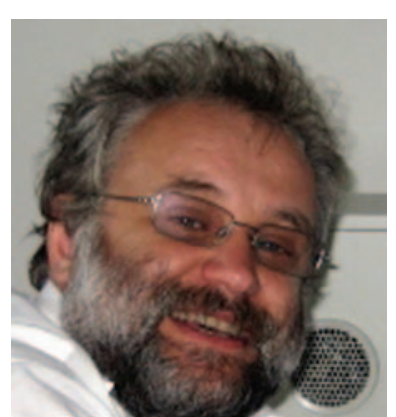

Andrei L. Tchougréeff is a principal scientist at the Division of Electrochemistry of the Department of Chemistry of the Lomonosov Moscow State University (MSU) and also senior scientist at RWTH Aachen University. He studied chemistry at the MSU and received his Master in 1982. His Ph.D. in chemical physics at the Karpov Institute (Moscow) defended in 1989 was devoted to the physics of conducting polymers. After a postdoctoral period spent in the USA, France and Germany between 1991 and 1995, he rejoined the Karpov Institute and habilitated (DSc) in 2004. In 2006, he joined the MSU, and since 1998 he has been organizing the prestigious V. A. Fock Meetings on Quantum and Computational Chemistry in Russia. His main research activities focus on the study of electronic structure of complex molecular systems with use of the group-functions approximation and on developing theoretical methods and computer codes suitable for that purpose.

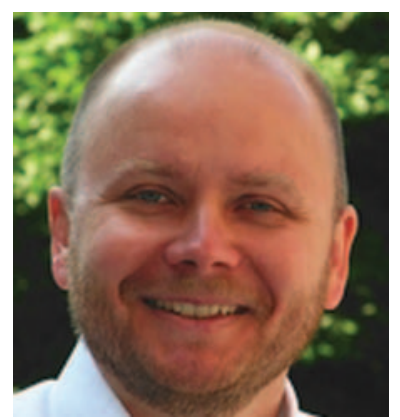

Richard Dronskowski born in 1961, studied chemistry and physics at Münster University (Germany) and received his doctorate from the Technical University of Stuttgart (Germany) in 1990; the dissertation dealt with condensed clusters in oxides and arsenides of molybdenum. After a one-year stay as a scientific visitor at Cornell University and a few more years as a senior scientist at the Max-Planck-Institute for Solid State Research in Stuttgart, he achieved his habilitation, covering the synthesis and electronic-structure theory of low-valent indium bromides, in Dortmund in 1995. In 1996 he went to RWTH Aachen University where he is currently holding the Chair of Solid-State and Quantum Chemistry. His interests lie in synthetic solid-state chemistry (nitrides, carbodiimides, guanidinates, magnetic intermetallics etc.), in high-resolution neutron diffraction, and in the quantum chemistry of the solid state. Various problems of electronic structure, magnetism, chemical bonding, phase prediction, and ab initio thermochemistry are being studied. 


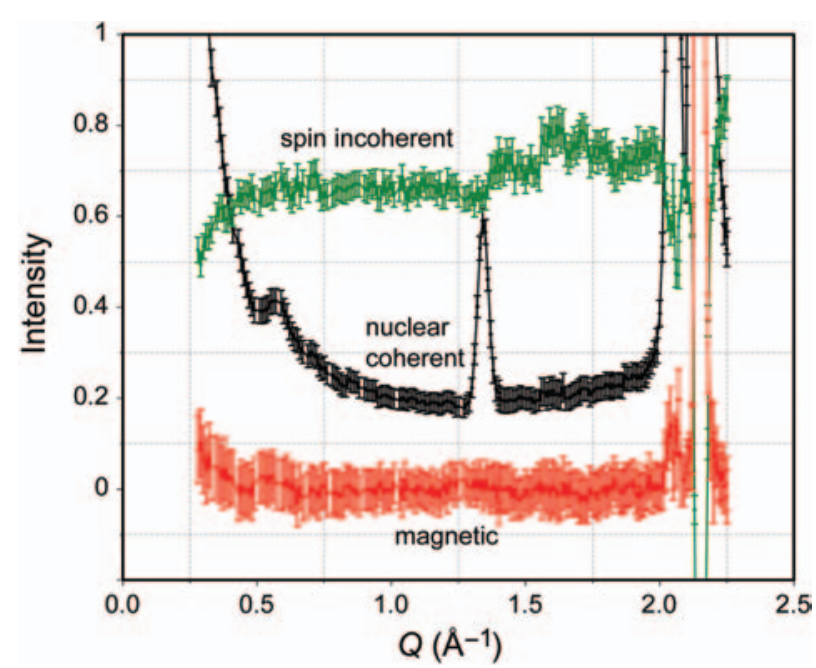

Fig. 2. Diffractional signal of CUNCN at $4.6 \mathrm{~K}$ measured with the DNS spectrometer (FRM II, Garching) using polarized neutrons. Reprinted with permission from [10], H. Xiang, et al., J. Phys. Chem. C 113, 18891 (2009). (C) 2009, American Chemical Society.

Now, the RVB treatment of the finite system exemplified by benzene is extended to infinite $\mathrm{CuNCN}^{13}$ by assuming the antiferromagnetic Heisenberg form of the Hamiltonian for the unpaired electrons residing in the $d^{9}$-shells of $\mathrm{Cu}^{2+}$. The corresponding exchange interactions $J_{c}, J_{a}$, and $J_{a c}$ are shown in Figure 1 and run along the crystallographic ac plane so that the Hamiltonian takes the form

$$
H=\sum_{r, \tau} J_{\tau} S_{r} S_{r+\tau}
$$

Here the summation over $r$ extends to all spins residing in the $\mathrm{Cu}^{2+}$ ions, that over $\tau$ to all neighbors of the given spin. The interactions in the $b$ direction (normal to the $a c$ planes) are neglected here. In the case of an infinite system, the wave function of the $d$-electrons (similar to the case of benzene in the $\pi$-approximation) is a linear combination of the products of the allowed pairings between the individual spins; note that all spins are paired as given by Eq. (1). The specific value of the amplitude of each such product must in principle be determined variationally to provide the minimum of the mean energy,

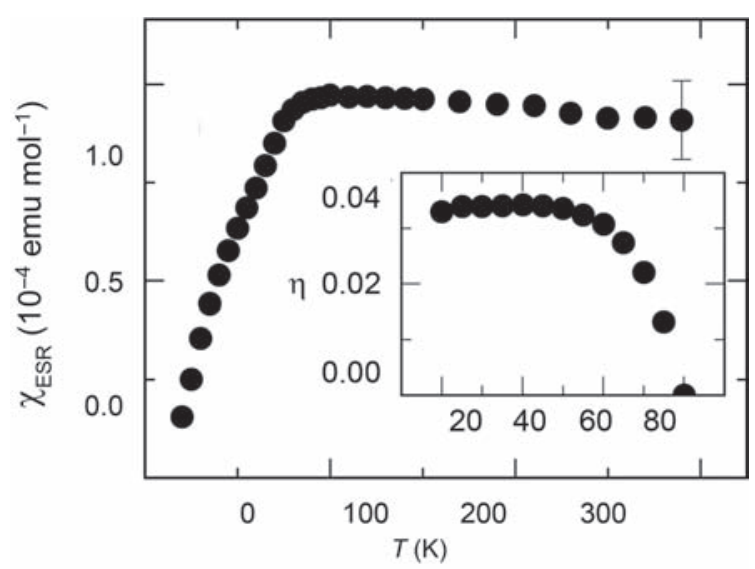

Fig. 3. The temperature course of the magnetic susceptibility and of the energy gap (inset) of CuNCN.

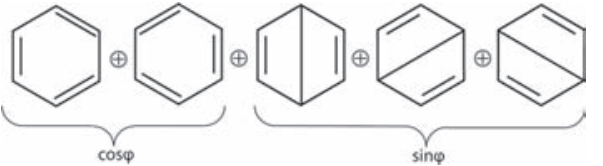

Fig. 4. Pictorial representation of the RVB state of the benzene molecule.

but this approach is unfeasible for the infinite system. Instead, the mean-field RVB approximation ${ }^{14}$ effectively parameterizes the required amplitudes of the individual products depicted in Figure 5 through so-called RVB order parameters $\zeta_{c}, \zeta_{a}$, and $\zeta_{a c}$. They are of nonvanishing value, correspond to the pairings extending in the respective direction in the crystal and serve as the cofactors of the respective exchange couplings in the Hamiltenian.

It is clear from the very beginning that in the RVB state one does not expect any magnetic neutron scattering since (although the spins are somehow ordered in the RVB state) the type of order is different from what is observed in a neutron experiment: we would then talk of the so-called off-diagonal long-range order in which the spin-spin correlation function whose divergence is ultimately detected in the (polarized) neutron scattering does not diverge. Another feature characteristic for the RVB states in the infinite systems is the rather peculiar spectrum of the related quasiparticles: spinons, possessing the fermion statistics, like electrons, but bearing only spin $1 / 2$ and no charge. ${ }^{15}$ For the proposed $c$ - $a$-ca model of $\mathrm{CuNCN}$ the quasiparticle spectrum has the form

$$
E_{k}= \pm \sqrt{A^{2} \cos ^{2} k_{a}+C^{2} \cos ^{2} k_{c}+4 B^{2} \cos ^{2} k_{a} \cos ^{2} k_{c}}
$$

where $k, k_{a}, k_{c}$ are, respectively, the two-dimensional wave vector (in the $a c$ plane) and its two components corresponding to the quasiparticles propagating in the $a$ and $c$ crystallographic directions. The spectral parameters $A, B$, and $C$ are given by:

$$
A=3 J_{a} \zeta_{a} \quad B=3 J_{a c} \zeta_{a c} \quad C=3 J_{c} \zeta_{c}
$$
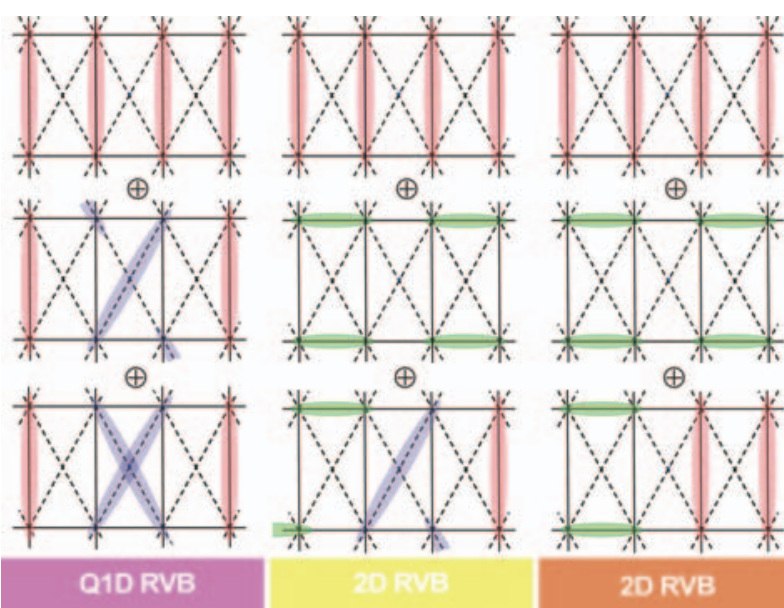

Fig. 5. Pictorial representation of the characteristic spin-pairings (valence bonds) contributing to various RVB states involved in the explanation of CuNCN. Adapted with permission of IOP from [13], A. L. Tchougréeff and R. Dronskowski, J. Phys.: Cond. Matt. 25, 435602 (2013). (c) 2013, All rights reserved. 
Depending on what order parameters $\left(\zeta_{\tau}\right.$ 's or, equivalently, the parameters $A, B$, and $C$ ) are vanishing, the quasiparticle spectrum manifests several largely different regimes as well as a very rich variety of magnetic behaviors. If all three order parameters become zero, the quasiparticle energy disappears as well which corresponds to a Curie paramagnetic behavior. The latter, however, is not observed in CuNCN. In other words, ${ }^{13}$ this must be a phase existing at very high temperature, presumably above the decomposition point of CuNCN. The phase where only $\zeta_{a c}(B)$ is nonvanishing exhibits a quasiparticle spectrum with two lines of nodes where the spinons have vanishing energy, hence producing the paramagnetic susceptibility logarithmically divergent at zero temperature. This theoretically predicted behavior is hardly distinguishable from the Curie paramagnetism and is not observed experimentally either.

Further RVB phases show even more peculiar forms of the quasiparticle spectrum. If only one of the order parameters $\zeta_{a}$ or $\zeta_{c}$ is nonvanishing, the spectrum is gapless for spinons propagating in the direction corresponding to this order parameter. In this case, the non-zero spectral parameters ( $A$ or $C$, respectively) play the role of the width of the quasiparticle band. These phases are fairly one-dimensional. If (in addition to either $\zeta_{a}$ or $\zeta_{c}$ ) the order parameter $\zeta_{a c}$ (respectively, $B$ ) is also nonvanishing, some dispersion appears in the direction transversal to that of the gapless spinon propagation. These states are therefore quasi-onedimensional in character. The overall bandwidth becomes either $\sqrt{C^{2}+4 B^{2}}$ or $\sqrt{A^{2}+4 B^{2}}$. Graphs of the corresponding spinon dispersion law and the quasiparticle density of states $(\mathrm{qDoS})$ are shown in Figure 6 (upper row); the nonvanishing spectral parameters $(A$ or $C)$ correspond to the saddle points in the dispersion law and thus mark the position of the logarithmic peak in the qDoS; note that, for the true one-dimensional state, the divergence of the qDoS on the ceiling of the quasiparticle band is of a power type. In the vicinity of the nodal lines the spinon energy is linearly dependent on $k_{a, c} \pm(\pi / 2)$ and the qDoS is thus finite at zero energy. This naturally produces the Pauli-like (temperatureindependent) paramagnetic form of the temperature dependence of the magnetic susceptibility observed at the higher temperature (above $100 \mathrm{~K}$ ).

If both order parameters $\zeta_{a}$ and $\zeta_{c}$ (and the spectral parameters $A$ or $C$ ) are nonvanishing, the spinon dispersion law has no nodal lines anymore but four nodal points at $( \pm(\pi / 2), \pm(\pi / 2))$ with vanishing spinon energies and a linear dependence on the wave vector in their vicinity. The dispersion law also contains the saddle points-so-called pseudogaps-which are now the meaning of the spectral parameters $A$ and $C$. These are the most characteristic features of the dispersion law for the two-dimensional RVB states of the $c-a-c a$ model. Accordingly, the qDoS in the two-dimensional state develops two logarithmic singularities at the pseudogap energies $A$ and $C$. Another interesting feature of the $\mathrm{qDoS}$ in the two-dimensional states is its linear dependence on the quasiparticle energy at the low energy, i.e., well below the smaller pseudogap. The corresponding graphs are shown in the middle and lower rows of Figure 6.

The magnetic properties in the two-dimensional phases are predetermined by the features of the corresponding qDoS. One may very well expect that the lower pseudogap takes part of the activation energy in the Arrhenius fit of the susceptibility. The relatively narrow energy range where the $\mathrm{qDoS}$ is linear with respect to the energy manifests only at very low temperature. The numerical calculation of the magnetic susceptibility

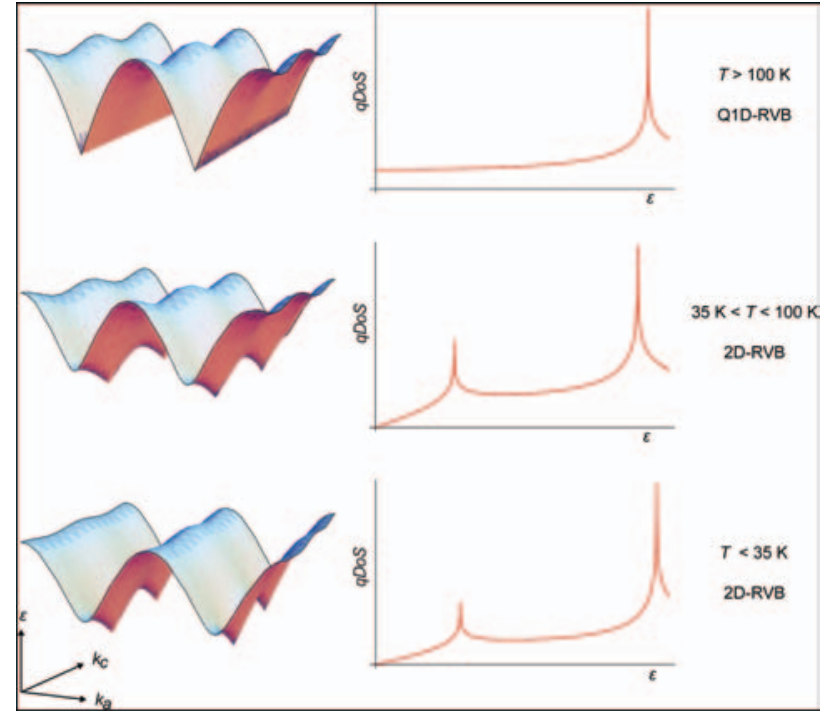

Fig. 6. Characteristics of three RVB phases of CuNCN involved in the explanation of the temperature course of the magnetic susceptibility and of the $a$ and $c$ lattice parameters. Reprinted with permission from [20], P. Jacobs, et al., J. Chem. Phys. 139, 224707 (2013). @ 2013, AIP Publishing LLC.

using the qDoS shown in Figure 6 then arrives at a satisfactory result. ${ }^{13}$ Here one has to allow for the so far unknown temperature dependence of the order parameters $\left(\zeta_{\tau}\right.$ 's or, equivalently, $A, B$, and $C)$. With this reservation the agreement between the theory and experiment is astonishingly good. The limiting values of the spectral parameters extracted from the experimental course of the magnetic susceptibility are: $C_{0}=1250 \mathrm{~K}, A_{0}=140 \mathrm{~K}$, and the temperature dependence of $A$ is interpolated by:

$$
A=A_{0}\left(1-\frac{T}{T_{\text {crit }}}\right)^{\beta}
$$

At this point we see that the hypothesis of the quantum spin-liquid (RVB) states in CuNCN explains, at least semiquantitatively, two key facts of the CuNCN physics: two temperature regimes of the susceptibility and the absence of the magnetic scattering of neutrons, namely, the absence of the magnetic diagonal long-range order. Theoretically, however, there are other possibilities as well: for example, the spin-Peierls scenario ${ }^{16}$ for $\mathrm{CuNCN}$, equivalent to the bond-order wave state, ${ }^{17,}{ }^{18}$ would do it equally well. If there were a spin-Peierls case, it would also explain the above two key features of $\mathrm{CuNCN}$ : the activation dependence of susceptibility due to opening of the spin-Peierls gap and the absence of the magnetic long-range order visible in the neutron scattering due to formation of $\mathrm{Cu}-\mathrm{Cu}$ spin pairings. In the spin-Peierls state, the spins would also be paired as they are in the RVB state (and as shown in Fig. 5) with the major difference that the pairings appear in a regular (e.g., alternating) fashion. That is to say that each odd or each even pair of spins in arbitrary direction would be paired, thereby producing a variety of spin-Peierls phases. Nonetheless, due to the regular distribution of pairings in the lattice, the spin-Peierls state must exhibit structure manifestations through the corresponding alternation of the interatomic separations which reflect the alternation of pairings. ${ }^{16}$ This immediately asks for structural methods to distinguish between the two pairing scenarios: the spin-liquid 
(RVB) and spin-Peierls case. If the observed switch between the Pauli and Arrhenius regimes of the temperature dependence of the magnetic susceptibility were to go back to a transition to the spin-Peierls state, it would be accompanied by an appearing superstructure reflecting the corresponding pairings in the interatomic $\left(\mathrm{Cu}^{2+}-\mathrm{Cu}^{2+}\right)$ distances.

We note that the transitions between the RVB (spin-liquid) states also lead to subtle structural changes, however. ${ }^{13}$ Namely, the temperature variation of the lattice parameters can be related to the temperature-dependent pseudogaps $A$ and $C$ by

$$
\left(\begin{array}{c}
\delta a \\
\delta c
\end{array}\right)=\alpha T\left(\begin{array}{l}
a \\
c
\end{array}\right)+\left(\begin{array}{c}
\Lambda_{A, a} \\
\Lambda_{A, c}
\end{array}\right) A^{2}+\left(\begin{array}{c}
\Lambda_{C, a} \\
\Lambda_{C, c}
\end{array}\right) C^{2}
$$

The first term describes the background thermal expansion with the common coefficient $\alpha$ whereas the two terms quadratic

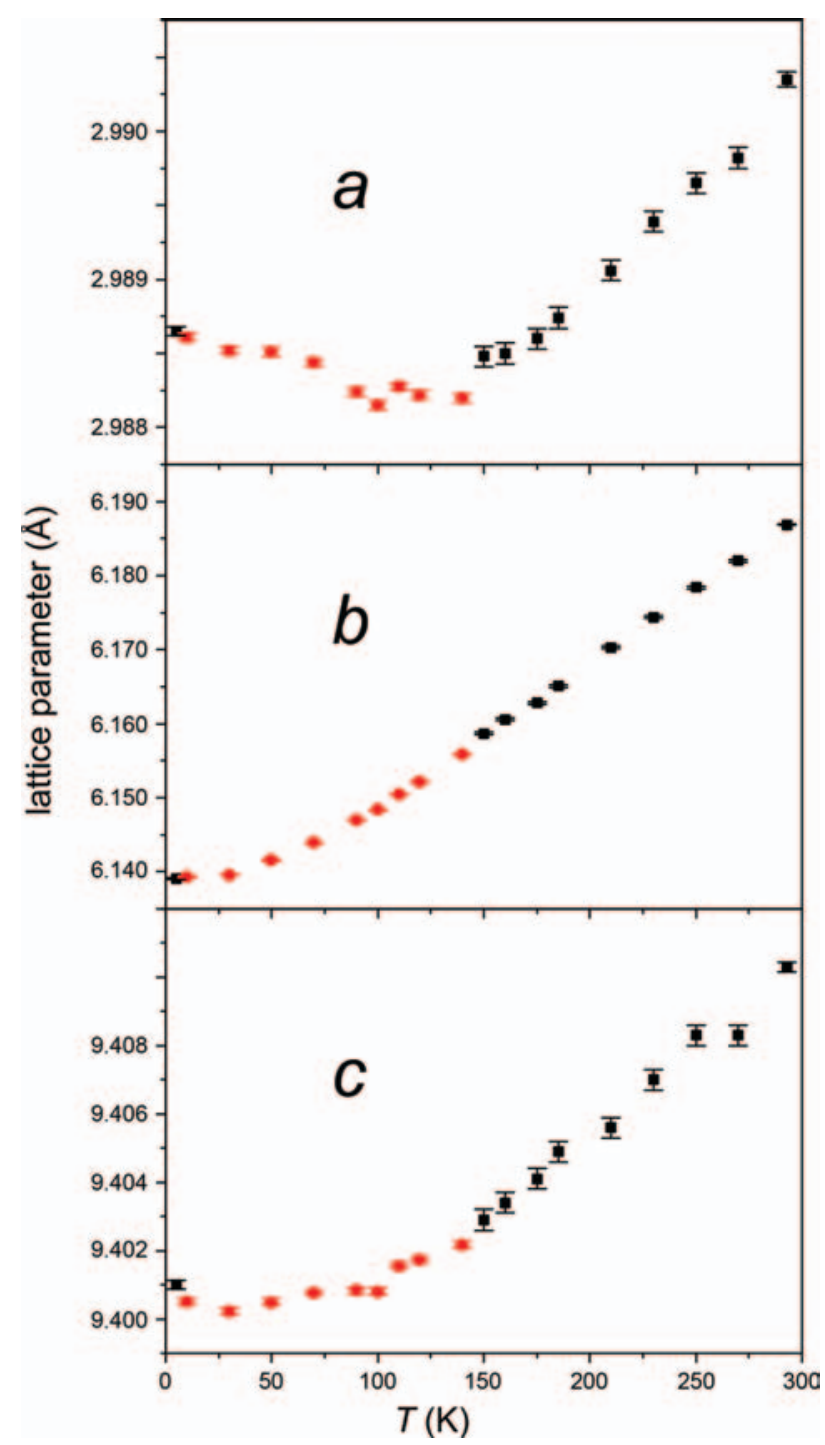

Fig. 7. Course of the lattice parameters $a, b$ and $c$ of $\mathrm{CuNCN}$ as a function of the temperature as extracted from $X$-ray synchrotron scattering; fastscan data in black, slow-scan data in red. For $b$, the standard deviations are smaller than the graphical symbol. Reprinted with permission from [19], A. L. Tchougréeff, et al., J. Phys. Chem. Lett. 3, 3360 (2012). (C) 2012, American Chemical Society. in the pseudogaps describe the effects of the involved RVB states upon the lattice parameters $a$ and $c$; the $\Lambda$ 's are combinations of the magnetic, elastic, and magnetostriction parameters and may be regarded as characteristic material constants. The explicit forms of the $\Lambda$ parameters have been derived ${ }^{13}$ by adding the elastic term

$$
\frac{1}{2}\left(\begin{array}{ll}
\delta a & \delta c
\end{array}\right)\left(\begin{array}{ll}
K_{a a} & K_{a c} \\
K_{a c} & K_{c c}
\end{array}\right)\left(\begin{array}{l}
\delta a \\
\delta c
\end{array}\right)
$$

to the Hamiltonian and modifying the exchange constants $J_{\tau}$ entering the Hamiltonian-spin-phonon coupling terms

$$
J_{\tau}=J_{\tau}^{(0)}+\sum_{\mu} J_{\tau, \mu}^{\prime} \rho_{\mu}
$$

where $\rho_{\mu}$ are the geometry variables $\delta a$ or $\delta c$.

The modelling ${ }^{13}$ of the magnetic susceptibility data ${ }^{12}$ is made under the assumption that the spectral parameter $C$ in the high-temperature (Pauli paramagnetic) and the Arrhenius paramagnetic phases reaches its limiting value $C_{0}$, so that the last term in Eq. (6) does not produce any temperature dependence. By contrast, the spectral parameter $A$ splits from zero at the critical temperature of the transition from the quasi-one-dimensional (high-temperature) to the two-dimensional (lower-temperature) RVB state; thus, the second term in Eq. (6) can produce a rather strong effect due to its strong (in fact, critical, see Eq. (5)) temperature dependence. Nevertheless, the structure manifestation of the transition between the spin-liquid (RVB) phases must be different from that of the spin-Peierls transition: it uniformly affects the lattice parameters, that is, leads to a change in size and shape of the unit cell, rather than the appearance of a superstructure.

The structure analysis has been carried out based on synchrotron X-ray ${ }^{19}$ and also using neutron scattering data. ${ }^{20}$ The results shown in Figure 7 consistently indicate a superposition between a more or less typical standard shrinking of the $\mathrm{CuNCN}$ crystal upon lowering the temperature (as described by the first term in Eq. (6)) plus some irregularities in the lattice parameters $a$ and $c$. Eventually, there is a distinct change of the sense of the temperature dependence for the lattice parameter $a$ : its decrease switches to an increase, and this happens right at the temperature at which the temperature regime of the magnetic susceptibility switches from the Pauli to the Arrhenius behavior. As depicted in

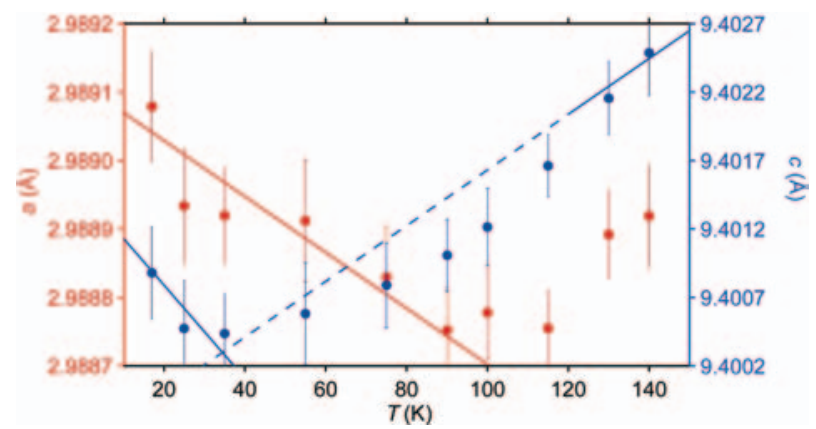

Fig. 8. The temperature dependence of the lattice parameters of CuNCN in the low-temperature region as extracted from neutron-diffraction data. The linear models serve to guide the eye. Reprinted with permission from [20], P. Jacobs, et al., J. Chem. Phys. 139, 224707 (2013). (c) 2013, AIP Publishing LLC. 
Table II. Sequence of RVB phase transitions in CuNCN.

\begin{tabular}{lcccc}
\hline Q1D-RVB & $T_{\text {crit }}$ & 2D-RVB transient & $T_{\text {crit }}$ & 2D-RVB ground state \\
\hline$B, C \neq 0 ; A=0$ & $\rightarrow$ & $A, B, C \neq 0$ & $\rightarrow$ & $A, C \neq 0 ; B=0$ \\
$\chi=$ const & $100 \mathrm{~K}$ & $\chi \propto \exp (-A / T)$ & $30 \mathrm{~K}$ & $\chi \propto \exp (-A / T)$ \\
$C^{\prime} \approx 0 ; C \approx C_{0}$ & \multicolumn{5}{c}{$C^{\prime} \approx 0 ; C \approx C_{0}$} & $A^{\prime} \approx 0 ; C^{\prime}<0$ \\
& $A \propto\left(1-T / T_{\text {crit }}\right)^{\beta}$ & \\
\hline
\end{tabular}

Figure 8 showing the details of the low-temperature range of the neutron scattering data, the switch of the sense of the temperature dependence of $a$ (and of the susceptibility temperature regime) is accompanied by an extra decrease of the lattice parameter $c$. This is a very remarkable feature since the theory predicts the signs of the constants $\Lambda_{A, a}$ and $\Lambda_{A, c}^{13}$ to be opposite. Thus, the structure effect of the transition from the (quasi-)one-dimensional to a two-dimensional RVB phase upon $a$ and $c$ must be opposite as well: an increase of the $a$ parameter requires an extra decrease of the $c$ parameter in addition to its background thermal shrinking, precisely as shown in Figure 8.

Even more intriguing events, however, evolve at lower temperatures when around $30 \mathrm{~K}$ the $c$ parameter changes the sign of its temperature slope. This very sign change of the $c$ versus $T$ dependency can be attributed to another transition between two RVB phases occurring in the $a c$ crystallographic planes of CuNCN. Namely, we have shown ${ }^{13,21}$ that the pseudogapped twodimensional RVB phase with the nonvanishing spectral parameters $A$ and $C$ exists in two versions: one in which only $A$ and $C$ are nonvanishing and another one where the spectral parameter $B$ is nonvanishing, too. The quasiparticle spectra of both phases are very similar: both have four nodal points and two pseudogaps, $A$ and $C$, in the spinon dispersion laws. Nevertheless, the qDoS's of the two phases differ: the one with the vanishing $B$ is concentrated in a narrower energy interval: its quasiparticle bandwidth is $\sqrt{A^{2}+C^{2}}$ and may be compared with $\sqrt{A^{2}+C^{2}+4 B^{2}}$. Also, the dispersion of the spinons in the $c$ direction seen along the ridges of the graphs in the middle and lower rows of Figure 6 is stronger for the phase with a nonvanishing $B$ (middle row). We could show ${ }^{21}$ that the two-dimensional RVB phase with three nonvanishing order or spectral parameters is transient in nature. It first installs at $100 \mathrm{~K}$ or slightly below because that is the opening of the pseudogap $A$ for the spinons propagating in the $a$ direction which leads to the phase with three nonvanishing spectral (order) parameters. Nonetheless, the lowest-energy RVB phase (presumably the RVB ground state) of the $c-a$-ca model is the two-dimensional RVB state with only two nonvanishing spectral parameters $(A$ and $C$ ) which persist down to zero temperature. Although the dispersion laws and qDoS's in these two phases are very similar, the physical properties differ. Specifically, the pseudogap $C$ manifests a temperature dependence in the lowtemperature $2 \mathrm{D}-\mathrm{RVB}$ phase; it starts decreasing ${ }^{13}$ with the temperature decrease from its limiting value $C_{0}$ almost reached in the Q1D-RVB and the transient 2D-RVB phases. The pseudogap $A$, by contrast, starts to develop in a critical manner as given by Eq. (5) at the critical temperature $T_{\text {crit }}=100 \mathrm{~K}$, and almost reaches its limiting value $A_{0}$ at the temperature of the conjectured second phase transition around $30 \mathrm{~K}$. Since the sign of the constant $\Lambda_{C, c}$ is negative ${ }^{13}$ as well, the slope of the temperature dependence of $c$ changes its sign as compared to the transient two-dimensional and quasi-one-dimensional RVB phases.
We also note that even within the achieved accuracy of the measurements there is not the slightest trace of any superstructure formation in either crystallographic direction, thereby excluding the proposed spin-Peierls state. While spin-Peierls alternatives to the here presented spin-liquid (RVB) picture may be considered intellectually inspiring, we conclude on the basis of the obtained structural information that nature clearly abandons this possibility.

To conclude, we may say with a high level of certainty that most probably the unusual physics of $\mathrm{CuNCN}$ is explained by the emergence of numerous spin-liquid states (or phases) stabilized due to frustration of the antiferromagnetic interactions $J_{c}$, $J_{a}$, and $J_{a c}$. Table II lists the sequence of the phase transitions conforming to the observed magnetic and structural behavior. Remarkably, the quasi-one-dimensional spin-liquid (RVB) phase of $\mathrm{CuNCN}$ exists between room temperature and down to $100 \mathrm{~K}$ where the two-dimensional spin-liquid phases install. Eventually, the spin-liquid phases of $\mathrm{CuNCN}$ are the highest-temperature spin liquids observed experimentally so far. Truly, CuNCN may be called a quantum material.

Unfortunately, our current knowledge of the precise magnitude of the magnetic, elastic, and magnetostriction parameters constituting the effective values of $\Lambda$ 's does not allow us to make more quantitative comparisons. The work on elucidating the exact form of the temperature dependence of the pseudogaps $A$ and $C$ is under way, which is going to precisely establish the value of the critical exponent $\beta$ and to give a final check of the used model for the magnetic susceptibility.

Acknowledgment: This work is partially supported by DFG and RFBR through grant No 14-03-00867.

\section{References and Notes}

1. C. Janiak, Dalton Trans. 2781 (2003).

2. M. Launay and R. Dronskowski, Z. Naturforsch. 60b, 437 (2005).

3. X. Liu, M. Krott, P. Müller, C. Hu, H. Lueken, and R. Dronskowski, Inorg. Chem. 44, 3001 (2005).

4. M. Krott, X. Liu, B. P. T. Fokwa, M. Speldrich, H. Lueken, and R. Dronskowski, Inorg. Chem. 46, 2204 (2007).

5. X. Liu, L. Stork, M. Speldrich, H. Lueken, and R. Dronskowski, Chem. Eur. J. 15, 1558 (2009).

6. M. Krott, A. Houben, P. Müller, W. Schweika, and R. Dronskowski, Phys. Rev. $B$ 80, 024117 (2009).

7. X. Tang, H. Xiang, X. Liu, M. Speldrich, and R. Dronskowski, Angew. Chem. Int. Ed. 49, 4738 (2010).

8. X.-H. Liu, R. Dronskowski, R. Glaum, and A. L. Tchougréeff, Z. Allg. Anorg. Chem. 636, 343 (2010).

9. A. L. Tchougréeff and R. Dronskowski, J. Phys. Chem. A 115, 4547 (2011)

10. H. Xiang, X. Liu, and R. Dronskowski, J. Phys. Chem. C 113, 18891 (2009).

11. X.-H. Liu, R. Dronskowski, R. K. Kremer, M. Ahrens, C.-D. Lee, and M.-H. Whangbo, J. Phys. Chem. C 112, 11013 (2008).

12. A. Zorko, P. Jeglič, A. Potočnik, D. Arčon, A. Balčytis, Z. Jagličić, X. Liu, A. L. Tchougréeff, and R. Dronskowski, Phys. Rev. Lett. 107, 047208 (2011).

13. A. L. Tchougréeff and R. Dronskowski, J. Phys.: Cond. Matt. 25, 435602 (2013).

14. M. Ogata and H. Fukuyama, Rep. Progr. Phys. 71, 036501 (2008)

15. K. I. Kugel and D. I. Khomskii, Sov. Phys. Usp. 25, 231 (1982).

16. A. I. Buzdin and L. N. Bulaevskiĭ, Sov. Phys. Usp. 23, 409 (1980).

17. A. E. Belinskii, A. L. Tchougréeff, and I. A. Misurkin, Teor. i Eksp. Khim. 25, 513 (1989), [in Russian]; Theor. and Exp. Chem. 25, 475 (1989), [in English].

18. H. Fukutome and M. Sasai, Prog. Theor. Phys. 67, 41 (1982)

19. A. L. Tchougréeff, X. Liu, P. Müller, W. van Beek, U. Ruschewitz, and R. Dronskowski, J. Phys. Chem. Lett. 3, 3360 (2012).

20. P. Jacobs, A. Houben, A. L. Tchougréeff, and R. Dronskowski, J. Chem. Phys. 139, 224707 (2013)

21. A. L. Tchougréeff and R. Dronskowski, Low Temp. Phys. 40, 92 (2014). 